\title{
Frames associated with shift invariant spaces on positive half line
}

\author{
Owais Ahmad \\ Department of Mathematics, \\ National Institute of Technology, \\ Srinagar-190006, \\ Jammu and Kashmir, India \\ email: siawoahmad@gmail.com
}

\author{
Mobin Ahmad \\ Department of Mathematics, \\ Faculty of Science, Jazan University, \\ Jazan -45142, Saudi Arabia \\ email: msyed@jazanu.edu.sa
}

\author{
Neyaz Ahmad \\ Department of Mathematics, \\ National Institute of Technology, \\ Srinagar-190006, \\ Jammu and Kashmir, India \\ email: neyaznit@yahoo.co.in
}

\begin{abstract}
In this paper, we introduce the notion of Walsh shift-invariant space and present a unified approach to the study of shift-invariant systems to be frames in $\mathrm{L}^{2}\left(\mathbb{R}^{+}\right)$. We obtain a necessary condition and three sufficient conditions under which the Walsh shift-invariant systems constitute frames for $\mathrm{L}^{2}\left(\mathbb{R}^{+}\right)$. Furthermore, we discuss applications of our main results to obtain some known conclusions about the Gabor frames and wavelet frames on positive half line.
\end{abstract}

\section{Introduction}

Shift-invariant spaces play an important role in modern analysis for the past two decades because of their rich underlying theory and their applications

2010 Mathematics Subject Classification: 42C15, 42C40, 43A70, 42A38, 41A17

Key words and phrases: shift-invariant space; Walsh wavelet frame; Gabor frame; WalshFourier transform 
in time frequency analysis, approximation theory, numerical analysis, digital signal and image processing and so on. A shift-invariant space serves as a universal model for sampling problem as it includes a large class of functions whether bandlimited or not by appropriately choosing a generator. The concept of shift-invariant subspace of $\mathrm{L}^{2}(\mathbb{R})$ was introduced by Helson [15]. In fact, he introduced range functions and used this notion to completely characterize shift invariant spaces. Later on, a considerable amount of research has been conducted using this framework in order to describe and characterize frames and bases of these spaces. For example, de Boor et al.[7] gave the general structure of these spaces in $L^{2}\left(\mathbb{R}^{n}\right)$ using the machinery of fiberization based on range functions. This has been further developed in the work of Ron and Shen [25] with the introduction of the technique of Gramians and dual Gramians. Bownik [8] gave a characterization of shift-invariant subspaces of $\mathrm{L}^{2}\left(\mathbb{R}^{\mathfrak{n}}\right)$ following an idea from Helson's book [15]. The invariance properties of shiftinvariant spaces under non-integer translations were completely characterized by Aldroubi et al.[4] and they showed that the principal shift-invariant spaces generated by a compactly supported function is not invariant under such translations. In [23], authors constructed $p$-frames for the weighted shift-invariant spaces and investigated their frame properties under some mild technical conditions on the frame generators. On the other side, the study of shift-invariant spaces and frames have been extended to locally compact Abelian groups in [9], nilpotent Lie groups in [11] and non-abelian compact groups in [24]. The results of Aldroubi et al.[4] were further generalized to the context of LCA groups by Anastasio et al.[5]. They provide necessary and sufficient conditions for an $\mathrm{H}$-invariant space to be $\mathrm{M}$-invariant space by means of range functions, where $\mathrm{H}$ is a countable uniform lattice in $\mathrm{G}$ and $\mathrm{M}$ is any closed subgroup of G containing $\mathrm{H}$. Shift-invariant spaces for local fields were first introduced and investigated by Ahmadi et al.[2]. More precisely, they studied shift-invariant spaces of $L^{2}(G)$, where $G$ is a locally compact abelian group, or in general a local field, with a compact open subgroup. The general results in Euclidean spaces to characterize tight frame generators for the shift-invariant subspaces was studied by Labate in [19]. Some applications of this general result are then obtained, among which are the characterization of tight wavelet frames and tight Gabor frames $[20,21]$. In his recent paper, Behera [6] showed that every closed shift-invariant subspace of $\mathrm{L}^{2}\left(\mathbb{R}^{+}\right)$is generated by the $\Lambda$-translates of a countable number of functions, where $K$ is the local field of positive characteristic and $\Lambda$ is the associated translation set.

In the framework of mathematical analysis and linear algebra, redundant representations are obtained by analysing vectors with respect to an overcom- 
plete system. Then the obtained vectors are interpreted using the frame theory as introduced by Duffin and Schaeffer [12] and recently studied at depth, see [10] and the compressive list of references therein. Most commonly used coherent/structured frames are wavelet, Gabor, and wave-packet frames which are a mixture type of wavelet and Gabor frames [10]. Frames provide a useful model to obtain signal decompositions in cases where redundancy, robustness, oversampling, and irregular sampling ploy a role. Today, the theory of frames has become an interesting and fruitful field of mathematics with abundant applications in signal processing, image processing, harmonic analysis, Banach space theory, sampling theory, wireless sensor networks, optics, filter banks, quantum computing, and medicine. Recall that a countable collection $\left\{f_{k}: k \in \mathbb{Z}\right\}$ in an infinite-dimensional separable Hilbert space $\mathcal{H}$ is called a frame if there exist positive constants $A$ and $B$ such that

$$
A\|f\|^{2} \leq \sum_{k \in \mathbb{Z}}\left|\left\langle f, f_{k}\right\rangle\right|^{2} \leq B\|f\|^{2}
$$

holds for every $f \in \mathcal{H}$ and we call the optimal constants $A$ and $B$ the lower frame bound and the upper frame bound, respectively. If we only require the second inequality to hold in (1), then $\left\{f_{k}: k \in \mathbb{Z}\right\}$ is called a Bessel collection. A frame is tight if $A=B$ in (1) and if $A=B=1$ it is called a Parseval frame or a normalized tight frame.

During last two decades there is a substantial body of work that has been concerned with the wavelet and Gabor frames on positive half line. Kozyrev $[16]$ found a compactly supported p-adic wavelet basis for $\mathrm{L}^{2}\left(\mathbb{Q}_{p}\right)$ which is an analog of the Haar basis. It turns out that these wavelets are eigenfunctions of some p-adic pseudodifferential operators in [18]. Such property used to solve padic pseudodifferential equations which are needed for some physical problems. Khrennikov et al. [17] developed a method to find explicitly the solution for a wide class of evolutionary linear pseudo-differential equations. Farkov [13] indicated several differences between the constructed wavelets in Walsh analysis and the classical wavelets, and characterized all compactly supported refinable functions on the Vilenkin group $G_{p}$ with $p \geq 2$. Manchanda et al. [22] introduced the vector-valued wavelet packets and obtained their properties and orthogonality formulas. Albeverio et al. [3] presented a complete characterization of scaling functions generating an p-MRA, suggested a method for constructing sets of wavelet functions, and proved that any set of wavelet functions generates a p-adic wavelet frame. Shah [27] constructed Gabor frame on positive half line and obtain necessary and sufficient conditions for Gabor frames in $\mathrm{L}^{2}\left(\mathbb{R}^{+}\right)$. More Recently, Zhang [28] characterize the shift-invariant 
Bessel sequences, frame sequences and Riesz sequences in $\mathrm{L}^{2}\left(\mathbb{R}^{+}\right)$and give a characterization of dual wavelet frames using Walsh-Fourier transform.

Motivated and inspired by the above work, we introduce the notion of Walsh shift-invariant spaces and establish some necessary and sufficient conditions under which shift-invariant systems become frames in $\mathrm{L}^{2}\left(\mathbb{R}^{+}\right)$. Furthermore, we use these results to give some necessary conditions and sufficient conditions for Gabor frames and wavelet frames on positive half line.

The paper is structured as follows. In Section 2, we give a brief introduction to Walsh-Fourier analysis including the definition of shift-invariant spaces on half line. In Section 3, we obtain a necessary condition for the shift-invariant system to be a frame for $\mathrm{L}^{2}\left(\mathbb{R}^{+}\right)$. In Section 4 , we establish sufficient conditions for shift-invariant systems to be frames. Sections 5 and 6 discusses applications of the our main results to Gabor frames and wavelet frames, respectively on positive half line.

\section{Preliminaries and shift-invariant spaces on posi- tive half line}

As usual, let $\mathbb{R}^{+}=[0,+\infty), \mathbb{Z}^{+}=\{0,1,2, \ldots\}$ and $\mathbb{N}=\mathbb{Z}^{+}-\{0\}$. Denote by $[x]$ the integer part of $x$. Let $p$ be a fixed natural number greater than 1 . For $x \in \mathbb{R}^{+}$and any positive integer $\boldsymbol{j}$, we set

$$
x_{j}=\left[p^{j} x\right](\bmod p), \quad x_{-j}=\left[p^{1-j} x\right](\bmod p),
$$

where $x_{j}, x_{-j} \in\{0,1, \ldots, p-1\}$. Clearly, $x_{j}$ and $x_{-j}$ are the digits in the $p$ expansion of $x$ :

$$
x=\sum_{j<0} x_{-j} p^{-j-1}+\sum_{j>0} x_{j} p^{-j}
$$

Moreover, the first sum on the right is always finite. Besides,

$$
[x]=\sum_{j<0} x_{-j} p^{-j-1}, \quad\{x\}=\sum_{j>0} x_{j} p^{-j}
$$

where $[x]$ and $\{x\}$ are, respectively, the integral and fractional parts of $x$.

Consider on $\mathbb{R}^{+}$the addition defined as follows:

$$
x \oplus y=\sum_{j<0} \zeta_{j} p^{-j-1}+\sum_{j>0} \zeta_{j} p^{-j}
$$


with $\zeta_{j}=x_{j}+y_{j}(\bmod p), j \in \mathbb{Z} \backslash\{0\}$, where $\zeta_{j} \in\{0,1, \ldots, p-1\}$ and $x_{j}, y_{j}$ are calculated by (2). Clearly, $[x \oplus y]=[x] \oplus[y]$ and $\{x \oplus y\}=\{x\} \oplus\{y\}$. As usual, we write $z=x \ominus y$ if $z \oplus y=x$, where $\ominus$ denotes subtraction modulo $p$ in $\mathbb{R}^{+}$.

Let $\varepsilon_{p}=\exp (2 \pi i / p)$, we define a function $r_{0}(x)$ on $[0,1)$ by

$$
r_{0}(x)= \begin{cases}1, & \text { if } x \in[0,1 / p) \\ \varepsilon_{p}^{\ell}, & \text { if } x \in\left[\ell p^{-1},(\ell+1) p^{-1}\right), \quad \ell=1,2, \ldots, p-1 .\end{cases}
$$

The extension of the function $r_{0}$ to $\mathbb{R}^{+}$is given by the equality $r_{0}(x+1)=$ $r_{0}(x), \forall x \in \mathbb{R}^{+}$. Then, the system of generalized Walsh functions $\left\{w_{m}(x)\right.$ : $\left.\mathrm{m} \in \mathbb{Z}^{+}\right\}$on $[0,1)$ is defined by

$$
w_{0}(x) \equiv 1 \quad \text { and } \quad w_{m}(x)=\prod_{j=0}^{k}\left(r_{0}\left(p^{j} x\right)\right)^{\mu_{j}}
$$

where $m=\sum_{j=0}^{k} \mu_{j} p^{j}, \mu_{j} \in\{0,1, \ldots, p-1\}, \mu_{k} \neq 0$. They have many properties similar to those of the Haar functions and trigonometric series, and form a complete orthogonal system. Further, by a Walsh polynomial we shall mean a finite linear combination of generalized Walsh functions. For $x, y \in \mathbb{R}^{+}$, let

$$
\chi(x, y)=\exp \left(\frac{2 \pi i}{p} \sum_{j=1}^{\infty}\left(x_{j} y_{-j}+x_{-j} y_{j}\right)\right)
$$

where $x_{j}, y_{j}$ are given by equation (2).

We observe that

$$
\chi\left(x, \frac{\mathrm{m}}{\mathrm{p}^{\mathrm{n}}}\right)=\chi\left(\frac{x}{\mathrm{p}^{\mathrm{n}}}, \mathrm{m}\right)=w_{\mathrm{m}}\left(\frac{x}{\mathrm{p}^{\mathrm{n}}}\right), \quad \forall x \in\left[0, \mathrm{p}^{\mathrm{n}}\right), \mathrm{m}, \mathrm{n} \in \mathbb{Z}^{+},
$$

and

$$
\chi(x \oplus y, z)=\chi(x, z) \chi(y, z), \quad \chi(x \ominus y, z)=\chi(x, z) \overline{\chi(y, z)},
$$

where $x, y, z \in \mathbb{R}^{+}$and $x \oplus y$ is p-adic irrational. It is well known that systems $\{\chi(\alpha, .)\}_{\alpha=0}^{\infty}$ and $\{\chi(\cdot, \alpha)\}_{\alpha=0}^{\infty}$ are orthonormal bases in $\mathrm{L}^{2}[0,1)$ (See $[14,26]$ ).

The Walsh-Fourier transform of a function $f \in \mathrm{L}^{1}\left(\mathbb{R}^{+}\right) \cap \mathrm{L}^{2}\left(\mathbb{R}^{+}\right)$is defined by

$$
\widehat{f}(\xi)=\int_{\mathbb{R}^{+}} f(x) \overline{\chi(x, \xi)} d x,
$$


where $\chi(\chi, \xi)$ is given by $(3)$. The Walsh-Fourier operator $\mathcal{F}: L^{1}\left(\mathbb{R}^{+}\right) \cap$ $\mathrm{L}^{2}\left(\mathbb{R}^{+}\right) \rightarrow \mathrm{L}^{2}\left(\mathbb{R}^{+}\right), \mathcal{F} \mathbf{f}=\widehat{\mathrm{f}}$, extends uniquely to the whole space $\mathrm{L}^{2}\left(\mathbb{R}^{+}\right)$. The properties of the Walsh-Fourier transform are quite similar to those of the classic Fourier transform (see $[14,26])$. In particular, if $f \in \mathrm{L}^{1}\left(\mathbb{R}^{+}\right) \cap \mathrm{L}^{2}\left(\mathbb{R}^{+}\right)$, then $\widehat{f} \in \mathrm{L}^{2}\left(\mathbb{R}^{+}\right)$and

$$
\|\hat{\mathrm{f}}\|_{\mathrm{L}^{2}\left(\mathbb{R}^{+}\right)}=\|f\|_{\mathrm{L}^{2}\left(\mathbb{R}^{+}\right)} .
$$

Moreover, if $f \in \mathrm{L}^{2}[0,1)$, then we can define the Walsh-Fourier coefficients of $f$ as

$$
\widehat{f}(n)=\int_{0}^{1} f(x) \overline{w_{n}(x)} d x .
$$

The series $\sum_{\mathfrak{n} \in \mathbb{Z}^{+}} \widehat{\mathrm{f}}(\mathfrak{n}) \boldsymbol{w}_{\mathfrak{n}}(x)$ is called the Walsh-Fourier series of $\mathrm{f}$. Therefore, from the standard $\mathrm{L}^{2}$-theory, we conclude that the Walsh-Fourier series of $f$ converges to $f$ in $L^{2}[0,1)$ and Parseval's identity holds:

$$
\|f\|_{2}^{2}=\int_{0}^{1}|f(x)|^{2} d x=\sum_{n \in \mathbb{Z}^{+}}|\widehat{f}(n)|^{2} .
$$

By p-adic interval $\mathrm{I} \subset \mathbb{R}^{+}$of range $\mathrm{n}$, we mean intervals of the form

$$
\mathrm{I}=\mathrm{I}_{\mathfrak{n}}^{\mathrm{k}}=\left[k \mathrm{p}^{-\mathrm{n}},(\mathrm{k}+1) \mathrm{p}^{-\mathrm{n}}\right), \quad \mathrm{k} \in \mathbb{Z}^{+} .
$$

The p-adic topology is generated by the collection of $p$-adic intervals and each $p$-adic interval is both open and closed under the p-adic topology (see $[14])$. The family $\left\{\left[0, p^{-j}\right): j \in \mathbb{Z}\right\}$ forms a fundamental system of the $p$-adic topology on $\mathbb{R}^{+}$. Therefore, the generalized Walsh functions $w_{j}(x), 0 \leq j \leq$ $p^{\mathfrak{n}}-1$, assume constant values on each $p$-adic interval $I_{n}^{k}$ and hence continuous on these intervals. Thus, $w_{j}(x)=1$ for $x \in I_{n}^{0}$.

Let $\mathcal{E}_{n}\left(\mathbb{R}^{+}\right)$be the space of $p$-adic entire functions of order $n$, that is, the set of all functions which are constant on all $p$-adic intervals of range $n$. Thus, for every $f \in \mathcal{E}_{n}\left(\mathbb{R}^{+}\right)$, we have

$$
f(x)=\sum_{k \in \mathbb{Z}^{+}} f\left(p^{-n} k\right) \chi_{I_{n}^{k}}(x), \quad x \in \mathbb{R}^{+} .
$$

Clearly each Walsh function of order up to $\mathrm{p}^{\mathrm{n}-1}$ belongs to $\mathcal{E}_{\mathrm{n}}\left(\mathbb{R}^{+}\right)$. The set $\mathcal{E}\left(\mathbb{R}^{+}\right)$of $p$-adic entire functions on $\mathbb{R}^{+}$is the union of all the spaces $\mathcal{E}_{n}\left(\mathbb{R}^{+}\right)$. It is clear that $\mathcal{E}\left(\mathbb{R}^{+}\right)$is dense in $\mathrm{L}^{\mathrm{p}}\left(\mathbb{R}^{+}\right), 1 \leq p<\infty$ and each function in $\mathcal{E}\left(\mathbb{R}^{+}\right)$is of compact support. Thus, we consider the following set of functions

$$
\mathcal{E}^{0}\left(\mathbb{R}^{+}\right)=\left\{f \in \mathcal{E}\left(\mathbb{R}^{+}\right): \widehat{f} \in L^{\infty}\left(\mathbb{R}^{+}\right) \text {and supp } f \subset \mathbb{R}^{+} \backslash\{0\}\right\} .
$$


For any $A \in \mathbb{R}^{+}$and $m, n \in \mathbb{Z}^{+}$, we define the following operators on $\mathrm{L}^{2}\left(\mathbb{R}^{+}\right)$ as:

$$
T_{n} f(x)=f(x \ominus n) ; E_{m} f(x)=\chi_{m}(x) f(x) ; D_{A} f(x)=\sqrt{A} f(A x) .
$$

Then for any $f \in L^{1}\left(\mathbb{R}^{+}\right) \cap L^{2}\left(\mathbb{R}^{+}\right)$, the following results can easily be verified:

$$
\begin{aligned}
& \mathcal{F}\left\{\mathrm{T}_{\mathrm{n}} \mathrm{f}(\mathrm{x})\right\}=\mathrm{E}_{-\mathrm{n}} \mathcal{F}\{\mathrm{f}(\mathrm{x})\} ; \mathcal{F}\left\{\mathrm{E}_{\mathrm{m}} \mathrm{f}(\mathrm{x})\right\}=\mathrm{T}_{\mathrm{m}} \mathcal{F}\{\mathrm{f}(\mathrm{x})\} ; \\
& \mathcal{F}\left\{\mathrm{D}_{\mathrm{A}^{\mathrm{j}}} \mathrm{f}(\mathrm{x})\right\}=\mathrm{D}_{\mathrm{A}^{-\mathrm{j}}} \mathcal{F}\{\mathrm{f}(\mathrm{x})\} .
\end{aligned}
$$

Definition 1 A closed subspace $\mathrm{S}$ of $\mathrm{L}^{2}\left(\mathbb{R}^{+}\right)$is called a Walsh shift invariant space if $T_{k} \varphi_{\alpha}(x) \in S$, for every $\varphi_{\alpha} \in S, k \in \mathbb{Z}^{+}$, and $\alpha \in \Lambda$, where $T_{k}$ is the translation operator and $\Lambda$ is a countable indexing set.

A closed shift-invariant subspace $\mathrm{S}$ of $\mathrm{L}^{2}\left(\mathbb{R}^{+}\right)$is said to be generated by $\Psi \subset$ $\mathrm{L}^{2}\left(\mathbb{R}^{+}\right)$if $\mathrm{S}=\overline{\operatorname{span}}\left\{\mathrm{T}_{\mathrm{k}} \psi_{\alpha}(\mathrm{x}):=\psi_{\alpha}(\mathrm{x} \ominus \mathrm{k}): \mathrm{k} \in \mathbb{Z}^{+}, \psi_{\alpha} \in \Psi\right\}$. The cardinality of a smallest generating set $\Psi$ for $\mathrm{S}$ is called the length of $\mathrm{S}$ which is denoted by $|\mathrm{S}|$. If $|\mathrm{S}|=$ finite, then $\mathbf{S}$ is called a finite Walsh shift-invariant space (FSI) and if $|\mathrm{S}|=1$, then $\mathrm{S}$ is called a principal Walsh shift-invariant space (PSI). Moreover, the spectrum of a Walsh shift-invariant space is defined to be

$$
\sigma(S)=\{\xi \in[0,1): \widehat{S}(\xi) \neq\{0\}\},
$$

where $\widehat{\mathbf{S}}(\xi)=\left\{\widehat{\varphi}_{\alpha}(\xi \oplus \mathrm{k}) \in \mathrm{l}^{2}\left(\mathbb{Z}^{+}\right): \varphi_{\alpha} \in \mathrm{S}, \mathrm{k} \in \mathbb{Z}^{+}, \alpha \in \Lambda\right\}$.

It is easy to verify that the system

$$
\Gamma=\left\{T_{k} \varphi_{\alpha}(x)=: \varphi_{\alpha}(x \ominus k): k \in \mathbb{Z}^{+}, \alpha \in \Lambda\right\},
$$

is a Walsh shift-invariant system with respect to lattice $\mathbb{Z}^{+}$, where $\varphi_{\alpha}(\mathrm{x}) \in$ $\mathrm{L}^{2}\left(\mathbb{R}^{+}\right)$.

Definition 2 The Walsh shift-invariant system $\Gamma$ defined by (11) is called shift-invariant frame if there exist constants $\mathrm{C}$ and $\mathrm{D}$ with $\mathrm{O}<\mathrm{C} \leq \mathrm{D}<\infty$ such that

$$
\mathrm{C}\|\varphi\|_{2}^{2} \leq \sum_{\alpha \in \Lambda} \sum_{n \in \mathbb{Z}^{+}}\left|\left\langle\varphi, \mathrm{T}_{\mathrm{n}} \varphi_{\alpha}\right\rangle\right|^{2} \leq \mathrm{D}\|\varphi\|_{2}^{2},
$$

holds for every $\varphi \in \mathrm{L}^{2}\left(\mathbb{R}^{+}\right)$. The largest constant $\mathrm{A}$ and the smallest constant $\mathrm{B}$ satisfying (12) are called the optimal lower and upper frame bounds, respectively. A frame is a tight frame if $\mathrm{A}$ and $\mathrm{B}$ are chosen so that $\mathrm{A}=\mathrm{B}$ and is a Parseval frame if $\mathrm{A}=\mathrm{B}=1$.

Since the set $\mathcal{E}\left(\mathbb{R}^{+}\right)$is dense in $\mathrm{L}^{2}\left(\mathbb{R}^{+}\right)$and is closed under the Fourier transform, the set $\mathcal{E}^{0}\left(\mathbb{R}^{+}\right)$defined by (9) is also dense in $\mathrm{L}^{2}\left(\mathbb{R}^{+}\right)$. Therefore, it is sufficient to prove that the Walsh shift-invariant system $\Gamma$ given by (11) is a frame for $\mathrm{L}^{2}\left(\mathbb{R}^{+}\right)$if the inequalities in (12) holds for all $\varphi \in \mathcal{E}^{0}\left(\mathbb{R}^{+}\right)$. 


\section{Necessary condition for Walsh shift invariant space to be frame for $\mathrm{L}^{2}\left(\mathbb{R}^{+}\right)$}

In this section, we shall study the necessary condition for the Walsh shiftinvariant system $\Gamma$ defined by (11) to be frame for $\mathrm{L}^{2}\left(\mathbb{R}^{+}\right)$.

Theorem 1 If the Walsh shift-invariant system $\Gamma$ defined by (11) is a frame for $\mathrm{L}^{2}\left(\mathbb{R}^{+}\right)$with bounds $\mathrm{A}$ and $\mathrm{B}$, then

$$
A \leq \mathrm{G}_{\Gamma}(\xi) \leq \mathrm{B}, \quad \text { a.e. } \xi \in \mathbb{R}^{+}
$$

where $\mathrm{G}_{\Gamma}(\xi)=\sum_{\alpha \in \Lambda}\left|\widehat{\varphi}_{\alpha}(\xi)\right|^{2}$.

Proof. Since the Walsh shift-invariant system $\Gamma$ is a frame for $\mathrm{L}^{2}\left(\mathbb{R}^{+}\right)$with bounds $A$ and $B$, we have

$$
\mathrm{A}\|\varphi\|_{2}^{2} \leq \sum_{\alpha \in \Lambda} \sum_{n \in \mathbb{Z}^{+}}\left|\left\langle\varphi, \mathrm{T}_{n} \varphi_{\alpha}\right\rangle\right|^{2} \leq \mathrm{B}\|\varphi\|_{2}^{2}, \quad \text { for all } \varphi \in \mathrm{L}^{2}\left(\mathbb{R}^{+}\right) .
$$

Notice that for all $\varphi \in \mathrm{L}^{2}\left(\mathbb{R}^{+}\right)$and $\mathrm{m} \in \mathbb{Z}^{+}$. By substituting $\varphi$ by $\mathrm{T}_{\mathrm{m}} \varphi$, equation (14) can be rewritten as

$$
\mathrm{A}\|\varphi\|_{2}^{2} \leq \sum_{\alpha \in \Lambda} \sum_{n \in \mathbb{Z}^{+}}\left|\left\langle\mathrm{T}_{\mathrm{m}} \varphi, \mathrm{T}_{\mathrm{n}} \varphi_{\alpha}\right\rangle\right|^{2} \leq \mathrm{B}\|\varphi\|_{2}^{2} .
$$

Or, equivalently

$$
\begin{aligned}
A\|\varphi\|_{2}^{2} \leq \int_{[0,1)} \sum_{\alpha \in \Lambda} \sum_{n \in \mathbb{Z}^{+}}\left|\left\langle T_{m} \varphi, T_{n} \varphi_{\alpha}\right\rangle\right|^{2} \mathrm{~d} x \leq \mathrm{B}\|\varphi\|_{2}^{2}, & \\
& \text { for all } \varphi \in \mathrm{L}^{2}\left(\mathbb{R}^{+}\right) .
\end{aligned}
$$

Since $\mathbb{R}^{+}=\bigcup_{\mathfrak{n} \in \mathbb{Z}^{+}}(\mathbb{T} \oplus \mathfrak{n})$ is a disjoint union,where $\mathbb{T}=[0,1)$. Therefore, it follows from the Plancherel theorem that 


$$
\begin{aligned}
\int_{\mathbb{T}} \sum_{\alpha \in \Lambda} \sum_{n \in \mathbb{Z}^{+}}\left|\left\langle T_{\mathrm{m}} \varphi, \mathrm{T}_{\mathrm{n}} \varphi_{\alpha}\right\rangle\right|^{2} \mathrm{~d} x & =\sum_{\alpha \in \Lambda} \sum_{\mathrm{n} \in \mathbb{Z}^{+}} \int_{\mathbb{T}}\left|\left\langle\varphi, \mathrm{T}_{\mathrm{n} \ominus \mathrm{m}} \varphi_{\alpha}\right\rangle\right|^{2} \mathrm{~d} x \\
& =\sum_{\alpha \in \Lambda} \int_{\mathbb{R}^{+}}\left|\left\langle\varphi, \mathrm{T}_{\mathrm{m}} \varphi_{\alpha}\right\rangle\right|^{2} \mathrm{~d} x \\
& =\sum_{\alpha \in \Lambda} \int_{\mathbb{R}^{+}}\left|\left\langle\widehat{\varphi},\left(\mathrm{T}_{\mathrm{m}} \varphi_{\alpha}\right)^{\wedge}\right\rangle\right|^{2} \mathrm{~d} x \\
& =\sum_{\alpha \in \Lambda} \int_{\mathbb{R}^{+}}\left|\left\langle\widehat{\varphi}, \mathrm{E}_{\ominus \mathrm{m}} \widehat{\varphi}_{\alpha}\right\rangle\right|^{2} \mathrm{~d} x \\
& =\sum_{\alpha \in \Lambda} \int_{\mathbb{R}^{+}}\left|\int_{\mathbb{R}^{+}} \widehat{\varphi}(\xi) \widehat{\widehat{\varphi}_{\alpha}(\xi)} w_{\mathrm{m}}(\xi) \mathrm{d} \xi\right|^{2} \mathrm{~d} x .
\end{aligned}
$$

Clearly, for all $\varphi \in \mathcal{E}^{0}\left(\mathbb{R}^{+}\right)$, we have $\widehat{\varphi}(\xi) \overline{\widehat{\varphi}_{\alpha}(\xi)} \in \mathrm{L}^{1}\left(\mathbb{R}^{+}\right) \cap \mathrm{L}^{2}\left(\mathbb{R}^{+}\right)$. Therefore, it follows from (16) and the Plancherel theorem that for all $\varphi \in \mathcal{E}^{0}\left(\mathbb{R}^{+}\right)$,

$$
\begin{aligned}
\int_{\mathbb{T}} \sum_{\alpha \in \Lambda} \sum_{n \in \mathbb{Z}^{+}}\left|\left\langle\mathrm{T}_{\mathrm{m}} \varphi, \mathrm{T}_{\mathrm{n}} \varphi_{\alpha}\right\rangle\right|^{2} \mathrm{~d} x & =\sum_{\alpha \in \Lambda} \int_{\mathbb{R}^{+}}\left|\left(\widehat{\varphi} \widehat{\widehat{\varphi}_{\alpha}}\right)^{\vee}(\xi)\right|^{2} \mathrm{~d} x \\
& =\sum_{\alpha \in \Lambda} \int_{\mathbb{R}^{+}}\left|\widehat{\varphi}(\xi) \overline{\widehat{\varphi}_{\alpha}}(\xi)\right|^{2} \mathrm{~d} \xi \\
& =\int_{\mathbb{R}^{+}}|\widehat{\varphi}(\xi)|^{2} \sum_{\alpha \in \Lambda}\left|\widehat{\varphi}_{\alpha}(\xi)\right|^{2} \mathrm{~d} \xi .
\end{aligned}
$$

Combining (15) and (17), we observe that for all $\varphi \in \mathcal{E}^{0}\left(\mathbb{R}^{+}\right)$, we have

$$
\mathrm{A}\|\varphi\|_{2}^{2} \leq \int_{\mathbb{R}^{+}}|\widehat{\varphi}(\xi)|^{2} \sum_{\alpha \in \Lambda}\left|\widehat{\varphi}_{\alpha}(\xi)\right|^{2} \mathrm{~d} \xi \leq \mathrm{B}\|\varphi\|_{2}^{2} .
$$

Making appropriate choices of $\varphi \in \mathcal{E}^{0}\left(\mathbb{R}^{+}\right)$in (18), we obtain

$$
A \leq G_{\Gamma}(\xi) \leq B, \quad \text { a.e. } \xi \in \mathbb{R}^{+} .
$$

Thus the proof of Theorem 1 is complete.

\section{Sufficient conditions for Walsh shift invariant space to be frame for $L^{2}\left(\mathbb{R}^{+}\right)$}

In this section, we derive three sufficient conditions for the Walsh shift-invariant system $\Gamma$ to be a frame for $\mathrm{L}^{2}\left(\mathbb{R}^{+}\right)$.

In order to prove our results, we need the following lemma. 
Lemma 1 Suppose that Walsh shift-invariant system $\Gamma$ is defined by (11). If $\varphi \in \mathcal{E}^{0}\left(\mathbb{R}^{+}\right)$and $\operatorname{ess} \sup \left\{\sum_{\alpha \in \Lambda}\left|\widehat{\varphi}_{\alpha}(\xi)\right|^{2}: \xi \in \mathbb{R}^{+}\right\}<\infty$, then

$$
\sum_{\alpha \in \Lambda} \sum_{n \in \mathbb{Z}^{+}}\left|\left\langle\varphi, T_{n} \varphi_{\alpha}\right\rangle\right|^{2}=\int_{\mathbb{R}^{+}}|\widehat{\varphi}(\xi)|^{2} \sum_{\alpha \in \Lambda}\left|\widehat{\varphi}_{\alpha}(\xi)\right|^{2} \mathrm{~d} \xi+R(\varphi),
$$

where

$$
\mathrm{R}(\varphi)=\sum_{\alpha \in \Lambda} \sum_{\mathrm{m} \in \mathbb{N}} \int_{\mathbb{R}^{+}} \widehat{\varphi}(\xi) \overline{\widehat{\varphi}_{\alpha}(\xi)} \widehat{\varphi}(\xi \oplus \mathrm{m}) \overline{\widehat{\varphi}_{\alpha}(\xi \oplus \mathrm{m})} \mathrm{d} \xi
$$

Furthermore, the iterated series in (20) is absolutely convergent.

Proof. By Parseval formula, we have

$$
\begin{aligned}
\sum_{n \in \mathbb{Z}^{+}}\left|\left\langle\varphi, T_{n} \varphi_{\alpha}\right\rangle\right|^{2} & =\sum_{n \in \mathbb{Z}^{+}}\left|\left\langle\widehat{\varphi},\left(\mathrm{T}_{n} \varphi_{\alpha}\right)^{\wedge}\right\rangle\right|^{2} \\
& =\sum_{n \in \mathbb{Z}^{+}}\left|\int_{\mathbb{R}^{+}} \widehat{\varphi}(\xi) \overline{\widehat{\varphi}_{\alpha}(\xi)} w_{n}(\xi) d \xi\right|^{2} \\
& =\sum_{n \in \mathbb{Z}^{+}} \int_{\mathbb{R}^{+}}\left\{\sum_{m \in \mathbb{Z}^{+}} \int_{[0,1)} \widehat{\varphi}(\xi \oplus m) \overline{\hat{\varphi}_{\alpha}(\xi \oplus m)} w_{n}(\xi \oplus m) d \xi\right\} \\
& \times \overline{\widehat{\varphi}(\xi)} \widehat{\varphi}_{\alpha}(\xi) \bar{w}_{n}(\xi) d \xi .
\end{aligned}
$$

Notice that $\widehat{\varphi}$ has compact support and $\mathcal{w}_{\mathrm{nm}} \equiv 1$ for all $\mathrm{m}, \mathrm{n} \in \mathbb{Z}^{+}$. Therefore, by the convergence theorem of Fourier series on $\mathbb{T}$, we obtain

$$
\sum_{n \in \mathbb{Z}^{+}}\left|\left\langle\varphi, T_{n} \varphi_{\alpha}\right\rangle\right|^{2}=\int_{\mathbb{R}^{+}} \overline{\widehat{\varphi}(\xi)} \widehat{\varphi}_{\alpha}(\xi)\left\{\sum_{m \in \mathbb{Z}^{+}} \widehat{\varphi}(\xi \oplus m) \overline{\hat{\varphi}_{\alpha}(\xi \oplus m)}\right\} d \xi .
$$

We claim that

$$
\sum_{\alpha \in \Lambda} \sum_{n \in \mathbb{Z}^{+}}\left|\left\langle\varphi, T_{n} \varphi_{\alpha}\right\rangle\right|^{2}=\sum_{\alpha \in \Lambda} \int_{\mathbb{R}^{+}}|\widehat{\varphi}(\xi)|^{2}\left|\widehat{\varphi}_{\alpha}(\xi)\right|^{2} \mathrm{~d} \xi+R(\varphi),
$$

hold for all $\varphi \in \mathcal{E}^{0}\left(\mathbb{R}^{+}\right)$. In fact, by (21), we have 


$$
\begin{aligned}
& \sum_{\alpha \in \Lambda} \sum_{n \in \mathbb{Z}^{+}}\left|\left\langle\varphi, T_{n} \varphi_{\alpha}\right\rangle\right|^{2} \\
& =\sum_{\alpha \in \Lambda} \int_{\mathbb{R}^{+}}\left\{|\widehat{\varphi}(\xi)|^{2}\left|\widehat{\varphi}_{\alpha}(\xi)\right|^{2}+\overline{\widehat{\varphi}(\xi)} \widehat{\varphi}_{\alpha}(\xi) \sum_{m \in \mathbb{Z}^{+}} \widehat{\varphi}(\xi \oplus \mathrm{m}) \overline{\widehat{\varphi}_{\alpha}(\xi \oplus \mathrm{m})}\right\} \\
& =\sum_{\alpha \in \Lambda} \int_{\mathbb{R}^{+}}|\widehat{\varphi}(\xi)|^{2}\left|\widehat{\varphi}_{\alpha}(\xi)\right|^{2} \mathrm{~d} \xi+\mathrm{R}(\varphi) .
\end{aligned}
$$

This is just (22). Finally, by the condition that $\operatorname{ess} \sup \left\{\sum_{\alpha \in \Lambda}\left|\widehat{\varphi}_{\alpha}(\xi)\right|^{2}: \xi \in \mathbb{R}^{+}\right\}$ $<\infty$, and by invoking Levi Lemma, we have

$$
\sum_{\alpha \in \Lambda} \sum_{n \in \mathbb{Z}^{+}}\left|\left\langle\varphi, \mathrm{T}_{\mathrm{n}} \varphi_{\alpha}\right\rangle\right|^{2}=\int_{\mathbb{R}^{+}}|\widehat{\varphi}(\xi)|^{2} \sum_{\alpha \in \Lambda}\left|\widehat{\varphi}_{\alpha}(\xi)\right|^{2} \mathrm{~d} \xi+\mathrm{R}(\varphi) .
$$

We now proceed to prove that the iterated series in (20) is absolutely convergent. Note that

$$
\left|\widehat{\varphi}_{\alpha}(\xi) \widehat{\varphi}_{\alpha}(\xi \oplus \mathrm{m})\right| \leq \frac{1}{2}\left[\left|\widehat{\varphi}_{\alpha}(\xi)\right|^{2}+\left|\widehat{\varphi}_{\alpha}(\xi \oplus \mathrm{m})\right|^{2}\right] .
$$

We have

$$
\begin{aligned}
|\mathrm{R}(\varphi)| & =\left|\sum_{\alpha \in \Lambda} \sum_{m \in \mathbb{N}} \int_{\mathbb{R}^{+}} \widehat{\varphi}(\xi) \overline{\widehat{\varphi}_{\alpha}(\xi)} \widehat{\varphi}(\xi \oplus \mathrm{m}) \overline{\widehat{\varphi}_{\alpha}(\xi \oplus \mathrm{m})} \mathrm{d} \xi\right| \\
\leq & \sum_{\alpha \in \Lambda} \sum_{\mathrm{m} \in \mathbb{N}} \int_{\mathbb{R}^{+}}\left|\widehat{\varphi}(\xi) \overline{\widehat{\varphi}_{\alpha}(\xi)} \widehat{\varphi}(\xi \oplus \mathrm{m}) \overline{\widehat{\varphi}_{\alpha}(\xi \oplus \mathrm{m})} \mathrm{d} \xi\right| \\
\leq & \frac{1}{2} \sum_{\alpha \in \Lambda} \sum_{m \in \mathbb{N}} \int_{\mathbb{R}^{+}}|\widehat{\varphi}(\xi) \widehat{\varphi}(\xi \oplus \mathrm{m})|\left|\widehat{\varphi}_{\alpha}(\xi)\right|^{2} \mathrm{~d} \xi \\
& \quad+\frac{1}{2} \sum_{\alpha \in \Lambda} \sum_{m \in \mathbb{N}} \int_{\mathbb{R}^{+}}|\widehat{\varphi}(\xi) \widehat{\varphi}(\xi \oplus \mathrm{m})|\left|\widehat{\varphi}_{\alpha}(\xi \oplus \mathrm{m})\right|^{2} \mathrm{~d} \xi .
\end{aligned}
$$

Hence it suffices to verify that the series

$$
\sum_{\alpha \in \Lambda} \sum_{m \in \mathbb{N}} \int_{\mathbb{R}^{+}}|\widehat{\varphi}(\xi) \widehat{\varphi}(\xi \oplus m)|\left|\widehat{\varphi}_{\alpha}(\xi)\right|^{2} d \xi
$$

is convergent. In fact,

$$
\begin{aligned}
& \sum_{\alpha \in \Lambda} \sum_{m \in \mathbb{N}} \int_{\mathbb{R}^{+}}|\widehat{\varphi}(\xi) \widehat{\varphi}(\xi \oplus m)|\left|\widehat{\varphi}_{\alpha}(\xi)\right|^{2} \mathrm{~d} \xi \\
& \quad \leq \operatorname{ess} \sup _{\xi \in \mathbb{R}^{+}} \sum_{\alpha \in \Lambda}\left|\widehat{\varphi}_{\alpha}(\xi)\right|^{2} \sum_{m \in \mathbb{N}} \int_{\mathbb{R}^{+}}|\widehat{\varphi}(\xi) \widehat{\varphi}(\xi \oplus m)| d \xi .
\end{aligned}
$$


Since $m \in \mathbb{N}$ and $\varphi \in \mathcal{E}^{0}\left(\mathbb{R}^{+}\right)$, hence, only finite terms of the iterated series in (23) are non-zero. Consequently, (23) becomes

$$
\begin{aligned}
\sum_{\alpha \in \Lambda} \sum_{m \in \mathbb{N}} \int_{\mathbb{R}^{+}} \mid & \left.\widehat{\varphi}(\xi) \widehat{\varphi}(\xi \oplus m)|| \widehat{\varphi}_{\alpha}(\xi)\right|^{2} \mathrm{~d} \xi \\
& \leq \operatorname{ess}^{\sup _{\xi \in \mathbb{R}^{+}}} \sum_{\alpha \in \Lambda}\left|\widehat{\varphi}_{\alpha}(\xi)\right|^{2} \mathrm{C}\|\widehat{\varphi}\|^{2}
\end{aligned}
$$

where $C$ is a constant. Using the assumption $\operatorname{ess} \sup _{\xi \in \mathbb{R}^{+}} \sum_{\alpha \in \Lambda}\left|\widehat{f}_{\alpha}(\xi)\right|^{2}<\infty$ and equation (24), it follows that the series (20) is absolutely convergent for all $f \in \mathcal{E}^{0}\left(\mathbb{R}^{+}\right)$. The proof of the lemma is completed.

To establish the first sufficient condition of shift-invariant frame for $\mathrm{L}^{2}\left(\mathbb{R}^{+}\right)$, we put

$$
\begin{aligned}
\underline{G}_{\Gamma} & =\operatorname{essinf}\left\{G_{\Gamma}(\xi): \xi \in \mathbb{R}^{+}\right\}, \quad \bar{G}_{\Gamma}=\operatorname{ess} \sup \left\{G_{\Gamma}(\xi): \xi \in \mathbb{R}^{+}\right\}, \\
\Theta_{f}(m) & =\operatorname{ess} \sup \left\{\sum_{\alpha \in \Lambda}\left|\widehat{\varphi}_{\alpha}(\xi) \widehat{\varphi}_{\alpha}(\xi \oplus \mathrm{m})\right|: \xi \in \mathbb{R}^{+}\right\},
\end{aligned}
$$

where $\mathrm{G}_{\Gamma}(\xi)$ is the same as the one in (13).

Theorem 2 Suppose $\left\{\varphi_{\alpha}(x): \alpha \in \Lambda\right\} \subset \mathrm{L}^{1}\left(\mathbb{R}^{+}\right) \cap \mathrm{L}^{2}\left(\mathbb{R}^{+}\right)$such that

$$
\begin{aligned}
& \mathrm{c}_{1}=\underline{\mathrm{G}}_{\Gamma}-\sum_{\mathrm{m} \in \mathbb{N}}\left[\Theta_{\varphi}(\mathrm{m}) \cdot \Theta_{\varphi}(\ominus \mathrm{m})\right]^{1 / 2}>0, \\
& \mathrm{D}_{1}=\overline{\mathrm{G}}_{\Gamma}+\sum_{\mathrm{m} \in \mathbb{N}}\left[\Theta_{\varphi}(\mathrm{m}) \cdot \Theta_{\varphi}(\ominus \mathrm{m})\right]^{1 / 2}<+\infty .
\end{aligned}
$$

Then $\left\{\mathrm{T}_{\mathrm{n}} \varphi_{\alpha}(\mathrm{x}): \mathrm{n} \in \mathbb{Z}^{+}, \alpha \in \Lambda\right\}$ is a frame for $\mathrm{L}^{2}\left(\mathbb{R}^{+}\right)$with bounds $\mathrm{C}_{1}$ and $\mathrm{D}_{1}$.

Proof. By Lemma 1 and (26), equation (19) holds, where

$$
\mathrm{R}(\varphi)=\sum_{\mathrm{m} \in \mathbb{N}} \int_{\mathbb{R}^{+}} \widehat{\varphi}(\xi) \overline{\widehat{\varphi}(\xi \oplus \mathrm{m})} \sum_{\alpha \in \Lambda} \overline{\widehat{\varphi}_{\alpha}(\xi)} \widehat{\varphi}_{\alpha}(\xi \oplus \mathrm{m}) \mathrm{d} \xi .
$$

Using the Cauchy-Schwarz inequality, the change of variables $\eta=\xi \oplus m$ 
and (27), we obtain

$$
\begin{aligned}
& |R(\varphi)| \leq \sum_{m \in \mathbb{N}}\left\{\int_{\mathbb{R}^{+}}|\widehat{\varphi}(\xi)|^{2}\left|\sum_{\alpha \in \Lambda} \overline{\widehat{\varphi}_{\alpha}(\xi)} \widehat{\varphi}_{\alpha}(\xi \oplus m)\right| d \xi\right\}^{1 / 2} \\
& \times\left\{\int_{\mathbb{R}^{+}}|\widehat{\varphi}(\xi \oplus m)|^{2}\left|\sum_{\alpha \in \Lambda} \overline{\widehat{\varphi}_{\alpha}(\xi)} \widehat{\varphi}_{\alpha}(\xi \oplus m)\right| d \xi\right\}^{1 / 2} \\
& =\sum_{m \in \mathbb{N}}\left\{\int_{\mathbb{R}^{+}}|\widehat{\varphi}(\xi)|^{2}\left|\sum_{\alpha \in \Lambda} \overline{\widehat{\varphi}_{\alpha}(\xi)} \widehat{\varphi}_{\alpha}(\xi \oplus m)\right| d \xi\right\}^{1 / 2} \\
& \times\left\{\int_{\mathbb{R}^{+}}|\widehat{\varphi}(\eta)|^{2}\left|\sum_{\alpha \in \Lambda} \overline{\widehat{\varphi}_{\alpha}(\eta \ominus m)} \widehat{\varphi}_{\alpha}(\eta)\right| d \eta\right\}^{1 / 2} \\
& \leq \int_{\mathbb{R}^{+}}|\widehat{\varphi}(\xi)|^{2} \mathrm{~d} \xi \sum_{\mathrm{m} \in \mathbb{N}}\left[\Theta_{\varphi}(\mathrm{m}) \cdot \Theta_{\varphi}(\ominus \mathrm{m})\right]^{1 / 2} \\
& \leq\|\varphi\|_{2}^{2} \sum_{m \in \mathbb{N}}\left[\Theta_{\varphi}(m) \cdot \Theta_{\varphi}(\ominus m)\right]^{1 / 2} \text {. }
\end{aligned}
$$

Consequently, it follows from equations (19), (25), (26) and (28) that

$$
\mathrm{C}_{1}\|\varphi\|_{2}^{2} \leq \sum_{\alpha \in \Lambda} \sum_{n \in \mathbb{Z}^{+}}\left|\left\langle\varphi, \mathrm{T}_{\mathrm{n}} \varphi_{\alpha}\right\rangle\right|^{2} \leq \mathrm{D}_{1}\|\varphi\|_{2}^{2}, \quad \text { for all } \varphi \in \mathcal{E}^{0}\left(\mathbb{R}^{+}\right)
$$

The proof of Theorem 2 is complete.

Remark 1 It is easy to see that

$$
\sum_{m \in \mathbb{N}} \Theta_{\varphi}(m)=\sum_{m \in \mathbb{N}} \Theta_{\varphi}(\ominus m)
$$

Set $\Theta_{\varphi}=\sum_{m \in \mathbb{N}} \Theta_{\varphi}(\mathrm{m})$. Then by (27) and the Cauchy-Schwarz inequality, we have

$$
|R(\varphi)| \leq \sum_{m \in \mathbb{N}}\left[\Theta_{f}(m) \cdot \Theta_{f}(\ominus m)\right]^{1 / 2}\|f\|_{2}^{2} \leq \Theta_{f}\|f\|_{2}^{2}
$$

As a consequence, the following second sufficient condition is obtained. 
Theorem 3 Suppose $\left\{\varphi_{\alpha}(x): \alpha \in \Lambda\right\} \subset \mathrm{L}^{1}\left(\mathbb{R}^{+}\right) \cap \mathrm{L}^{2}\left(\mathbb{R}^{+}\right)$such that

$$
\begin{aligned}
\mathrm{C}_{2} & =\underline{\mathrm{G}}_{\Gamma}-\Theta_{\mathrm{f}}>0, \\
\mathrm{D}_{2} & =\overline{\mathrm{G}}_{\Gamma}+\Theta_{\mathrm{f}}<+\infty .
\end{aligned}
$$

Then $\left\{\mathrm{T}_{\mathrm{n}} \varphi_{\alpha}(\mathrm{x}): \mathrm{n} \in \mathbb{Z}^{+}, \alpha \in \Lambda\right\}$ is a frame for $\mathrm{L}^{2}\left(\mathbb{R}^{+}\right)$with bounds $\mathrm{C}_{2}$ and $\mathrm{D}_{2}$.

The proof follows in the similar lines to that of Theorem 2.

Using a different estimation technique, we are in a position to position to provide the third sufficient condition for the Walsh shift-invariant system $\mathcal{W}(\varphi, \alpha, k)$ to be frame of $\mathrm{L}^{2}\left(\mathbb{R}^{+}\right)$as follows:

Theorem 4 Suppose $\left\{\varphi_{\alpha}(x): \alpha \in \Lambda\right\} \subset \mathrm{L}^{1}\left(\mathbb{R}^{+}\right) \cap \mathrm{L}^{2}\left(\mathbb{R}^{+}\right)$such that

$$
\begin{aligned}
& \mathrm{C}_{3}=\operatorname{ess} \inf _{\xi \in \mathbb{R}^{+}}\left[\sum_{\alpha \in \Lambda}\left|\widehat{\varphi}_{\alpha}(\xi)\right|^{2}-\sum_{\mathrm{m} \in \mathbb{N}}\left|\sum_{\alpha \in \Lambda} \overline{\widehat{\varphi}_{\alpha}(\xi)} \widehat{\varphi}_{\alpha}(\xi \oplus \mathrm{m})\right|\right]>0, \\
& \mathrm{D}_{3}=\operatorname{ess} \sup _{\xi \in \mathbb{R}^{+}}\left[\sum_{\mathrm{m} \in \mathbb{Z}^{+}}\left|\sum_{\alpha \in \Lambda} \overline{\hat{\varphi}_{\alpha}(\xi)} \widehat{\varphi}_{\alpha}(\xi \oplus \mathrm{m})\right|\right]<+\infty .
\end{aligned}
$$

Then $\left\{\mathrm{T}_{\mathrm{n}} \varphi_{\alpha}(\mathrm{x}): \mathrm{n} \in \mathbb{Z}^{+}, \alpha \in \Lambda\right\}$ is a frame for $\mathrm{L}^{2}\left(\mathbb{R}^{+}\right)$with bounds $\mathrm{C}_{3}$ and $\mathrm{D}_{3}$.

Proof. We estimate $R(\varphi)$ in (27) by another technique. Using the CauchySchwarz inequality, the change of variables $\eta=\xi \oplus \mathrm{m}$ and the Levi Lemma, we have

$$
\begin{aligned}
|R(\varphi)| \leq & \sum_{m \in \mathbb{N}}\left\{\int_{\mathbb{R}^{+}}|\widehat{\varphi}(\xi)|^{2}\left|\sum_{\alpha \in \Lambda} \overline{\widehat{\varphi}_{\alpha}(\xi)} \widehat{\varphi}_{\alpha}(\xi \oplus \mathrm{m})\right| \mathrm{d} \xi\right\}^{1 / 2} \\
& \times\left\{\int_{\mathbb{R}^{+}}|\widehat{\varphi}(\xi \oplus \mathrm{m})|^{2}\left|\sum_{\alpha \in \Lambda} \overline{\widehat{\varphi}_{\alpha}(\xi)} \widehat{\varphi}_{\alpha}(\xi \oplus \mathrm{m})\right| \mathrm{d} \xi\right\}^{1 / 2} \\
\leq & \left\{\int_{\mathbb{R}^{+}}|\widehat{\varphi}(\xi)|^{2} \sum_{\mathrm{m} \in \mathbb{N}}\left|\sum_{\alpha \in \Lambda} \overline{\widehat{\varphi}_{\alpha}(\xi)} \widehat{\varphi}_{\alpha}(\xi \oplus \mathrm{m})\right| \mathrm{d} \xi\right\}^{1 / 2} \\
& \times\left\{\int_{\mathbb{R}^{+}}|\widehat{\varphi}(\eta)|^{2} \sum_{\mathrm{m} \in \mathbb{N}}\left|\sum_{\alpha \in \Lambda} \overline{\widehat{\varphi}_{\alpha}(\eta)} \widehat{\varphi}_{\alpha}(\eta \ominus \mathrm{m})\right| \mathrm{d} \eta\right\}^{1 / 2} \\
= & \int_{\mathbb{R}^{+}}|\widehat{\varphi}(\xi)|^{2} \sum_{\mathrm{m} \in \mathbb{N}} \mid \sum_{\alpha \in \Lambda} \frac{\widehat{\varphi}_{\alpha}(\xi) \widehat{\varphi}_{\alpha}(\xi \oplus \mathrm{m}) \mid \mathrm{d} \xi .}{}
\end{aligned}
$$


Consequently, it follows from equations (19), (31), (32) and (33) that

$$
\mathrm{C}_{3}\|\varphi\|_{2}^{2} \leq \sum_{\alpha \in \Lambda} \sum_{n \in \mathbb{Z}^{+}}\left|\left\langle\varphi, \mathrm{T}_{n} \varphi_{\alpha}\right\rangle\right|^{2} \leq \mathrm{D}_{3}\|\varphi\|_{2}^{2}, \quad \text { for all } \varphi \in \mathcal{E}^{0}\left(\mathbb{R}^{+}\right) .
$$

The proof of Theorem 4 is complete.

\section{Walsh shift-invariant systems as Gabor frames on positive half line}

In this section, we apply Theorems 1, 2 and 4 to the Gabor systems and obtain some results on Gabor frames on local fields of positive characteristic.

Gabor systems are the collection of functions

$$
\mathcal{G}(\mathrm{g}, \mathrm{m}, \mathrm{n})=\left\{\mathrm{M}_{\mathrm{m}} \mathrm{T}_{\mathrm{n}} \mathrm{g}(\mathrm{x})=: w_{\mathrm{m}}(\mathrm{x}) \mathrm{g}(\mathrm{x} \ominus \mathrm{n}): \mathrm{m}, \mathrm{n} \in \mathbb{Z}^{+}\right\}
$$

which are built by the action of modulations and translations of a single $\mathrm{g} \in$ $\mathrm{L}^{2}\left(\mathbb{R}^{+}\right)$. If we interchange the role of the translation and modulation operators, we have the system

$$
\mathcal{G}^{\prime}(g, m, n)=\left\{T_{n} M_{m} g(x)=: w_{m}(x) g(x \ominus n): m, n \in \mathbb{Z}^{+}\right\} .
$$

It is immediate to see that the system $\mathcal{G}(\mathrm{g}, \mathrm{m}, \mathrm{n})$ is a frame of $\mathrm{L}^{2}\left(\mathbb{R}^{+}\right)$if and only if $\mathcal{G}^{\prime}(\mathrm{g}, \mathrm{m} . \mathrm{n})$ is a frame of $\mathrm{L}^{2}\left(\mathbb{R}^{+}\right)$, and the frame bounds are the same in the two cases. It is evident that Gabor system (35) is shift-invariant. So, the main results in Sections 3 and 4 can apply directly to the Gabor systems.

Setting $\Lambda=\left\{m: m \in \mathbb{Z}^{+}\right\}$, and for all $\alpha \in \Lambda$, we take $\varphi_{\alpha}=E_{m} g(x)$. Then the system $\Gamma(\varphi, \alpha, \mathrm{m})$ given by (11) reduces to the Gabor system $\mathcal{G}(\mathrm{g}, \mathrm{m}, \mathrm{n})$ defined by (34). Notice that for all $\alpha \in \Lambda$,

$$
\widehat{\varphi}_{\alpha}(\xi)=\widehat{g}(\xi \ominus \mathrm{m}) \text {. }
$$

Therefore, for all $n \in \mathbb{Z}^{+}$, we have

$$
\sum_{\alpha \in \Lambda} \overline{\widehat{\varphi}_{\alpha}(\xi)} \widehat{\varphi}_{\alpha}(\xi \oplus n)=\sum_{m \in \mathbb{Z}^{+}} \overline{\widehat{g}(\xi \ominus \mathrm{m})} \widehat{g}(\xi \ominus \mathrm{m} \oplus \mathrm{n}) .
$$

Using Theorems 1, 2 and 4, we obtain 
Theorem 5 If the Gabor system $\mathcal{G}(\mathrm{g}, \mathrm{m}, \mathrm{n})$ defined by (34) is a frame for $\mathrm{L}^{2}\left(\mathbb{R}^{+}\right)$with bounds $\mathrm{C}_{4}$ and $\mathrm{D}_{4}$, then

$$
\mathrm{C}_{4} \leq \sum_{\mathrm{m} \in \mathbb{Z}^{+}}|\widehat{\mathrm{g}}(\xi \ominus \mathrm{m})|^{2} \leq \mathrm{D}_{4} \text {, a.e. } \xi \in \mathbb{R}^{+}
$$

Theorem 6 Suppose $\mathrm{g} \in \mathrm{L}^{1}\left(\mathbb{R}^{+}\right) \cap \mathrm{L}^{2}\left(\mathbb{R}^{+}\right)$such that

$$
\begin{aligned}
& \mathrm{C}_{5}=\operatorname{ess} \inf _{\xi \in \mathbb{R}^{+}} \sum_{m \in \mathbb{Z}^{+}}|\widehat{g}(\xi \ominus m)|^{2}-\sum_{n \in \mathbb{N}}\left[\Theta_{g}(n) \cdot \Theta_{g}(\ominus n)\right]^{1 / 2}>0, \\
& D_{5}=\operatorname{ess} \sup _{\xi \in \mathbb{R}^{+}} \sum_{m \in \mathbb{Z}^{+}}|\widehat{g}(\xi \ominus m)|^{2}+\sum_{n \in \mathbb{N}}\left[\Theta_{g}(n) \cdot \Theta_{g}(\ominus n)\right]^{1 / 2}<+\infty,
\end{aligned}
$$

then $\left\{M_{m} T_{n} g(x): m, n \in \mathbb{Z}^{+}\right\}$is a Gabor frame for $\mathrm{L}^{2}\left(\mathbb{R}^{+}\right)$with bounds $\mathrm{C}_{5}$ and $\mathrm{D}_{5}$, where

$$
\Theta_{g}(n)=\operatorname{ess} \sup \left\{\sum_{m \in \mathbb{Z}^{+}}|\widehat{g}(\xi \ominus m) \widehat{g}(\xi \ominus m \oplus n)|: \xi \in \mathbb{R}^{+}\right\} .
$$

Theorem 7 Suppose $\mathrm{g} \in \mathrm{L}^{1}\left(\mathbb{R}^{+}\right) \cap \mathrm{L}^{2}\left(\mathbb{R}^{+}\right)$such that

$$
\begin{aligned}
& \mathrm{C}_{6}=\operatorname{ess} \inf _{\xi \in \mathbb{R}^{+}}\left\{\sum_{\mathrm{m} \in \mathbb{Z}^{+}}|\widehat{\mathrm{g}}(\xi \ominus \mathrm{m})|^{2}-\sum_{\mathrm{n} \in \mathbb{N}}\left|\sum_{\mathrm{m} \in \mathbb{Z}^{+}} \overline{\widehat{g}(\xi \ominus \mathrm{m})} \widehat{\mathrm{g}}(\xi \ominus \mathrm{m} \oplus \mathrm{n})\right|\right\}>0, \\
& \mathrm{D}_{6}=\operatorname{ess} \sup _{\xi \in \mathbb{R}^{+}}\left\{\sum_{n \in \mathbb{Z}^{+}}\left|\sum_{\mathrm{m} \in \mathbb{Z}^{+}} \overline{\widehat{g}(\xi \ominus \mathrm{m})} \widehat{\mathrm{g}}(\xi \ominus \mathrm{m} \oplus \mathrm{n})\right|\right\}<+\infty .
\end{aligned}
$$

Then $\left\{M_{m} T_{n} g(x): m, n \in \mathbb{Z}^{+}\right\}$is a Gabor frame for $\mathrm{L}^{2}\left(\mathbb{R}^{+}\right)$with bounds $\mathrm{C}_{6}$ and $\mathrm{D}_{6}$.

\section{Remark 2 Since}

$$
\left\langle\varphi, T_{n} M_{m} g\right\rangle=\left\langle\varphi^{\vee},\left(T_{n} M_{m} g\right)^{\vee}\right\rangle=\left\langle\varphi^{\vee}, T_{\ominus m} M_{n} g^{\vee}\right\rangle
$$

by the Plancherel Theorem, similarly to the case in the frequency domain, we able to present similar results in the time domain. They were omitted. 


\section{Walsh shift-invariant systems as wavelet frames on positive half line}

In this section, we apply Theorems 1, 2 and 4 to the wavelet systems and obtain some results on wavelet frames on positive half line.

For a given $\psi \in \mathrm{L}^{2}\left(\mathbb{R}^{+}\right)$, define the wavelet system

$$
\mathcal{W}(\psi, j, k)=\left\{\psi_{j, k}(x): j \in \mathbb{Z}, k \in \mathbb{Z}^{+}\right\}
$$

where $\psi_{j, k}(x)=p^{j / 2} \psi\left(p^{j} x \ominus k\right)$. In general, the wavelet system $\mathcal{W}(\psi, j, k)$ is not shift-invariant, and thus the main results in Sections 3 and 4 do not apply directly to a wavelet system. But we can use a quasi-wavelet system to investigate the wavelet system. The quasi-wavelet system generated by $\psi \in$ $\mathrm{L}^{2}\left(\mathbb{R}^{+}\right)$is defined by

$$
\widetilde{\mathcal{W}}(\widetilde{\psi}, j, k)=\left\{\widetilde{\psi}_{j, k}(x): j \in \mathbb{Z}, k \in \mathbb{Z}^{+}\right\}
$$

where

$$
\widetilde{\psi}_{j, k}(x)=\left\{\begin{aligned}
D_{A^{j}} T_{k} \psi(x)=A^{j / 2} \psi\left(A^{j} x \ominus k\right), & j \geq 0, k \in \mathbb{Z}^{+}, \\
A^{j / 2} T_{k} D_{A^{j}} \psi(x)=A^{j} \psi\left(A^{j}(x \ominus k)\right), & j<0, k \in \mathbb{Z}^{+} .
\end{aligned}\right.
$$

It is easy to see that the quasi-wavelet system is shift-invariant. There is some sort of equivalence between wavelet and quasi-wavelet systems. Indeed, Abdullah [1] proved in full generality the following result on positive half line.

Theorem 8 Let $\psi \in \mathrm{L}^{2}\left(\mathbb{R}^{+}\right)$. Then

(a) $\mathcal{W}(\psi, j, k)$ is a Bessel family if and only if $\widetilde{\mathcal{W}}(\widetilde{\psi}, j, k)$ is a Bessel family. Furthermore, their exact upper bounds are equal.

(b) $\mathcal{W}(\psi, j, k)$ is a frame for $\mathrm{L}^{2}\left(\mathbb{R}^{+}\right)$if and only if $\widetilde{\mathcal{W}}(\widetilde{\psi}, \mathrm{j}, \mathrm{k})$ is a frame for $\mathrm{L}^{2}\left(\mathbb{R}^{+}\right)$. Furthermore, their lower and upper exact bounds are equal.

For $\mathfrak{j} \in \mathbb{Z}^{+}$, let $\mathcal{N}_{j}$ denotes a full collection of coset representatives of $\mathbb{Z}^{+} / A^{j} \mathbb{Z}^{+}$, i.e.,

$$
\mathcal{N}_{j}=\left\{\begin{array}{rr}
\left\{0,1,2, \ldots, A^{j}-1\right\}, & j \geq 0 \\
\{0\}, & j<0 .
\end{array}\right.
$$

Then, $\mathbb{Z}^{+}=\bigcup_{n \in \mathcal{N}_{j}}\left(n \oplus A^{j} \mathbb{Z}^{+}\right)$, and for any distinct $n_{1}, n_{2} \in \mathcal{N}_{j}$, we have $\left(n_{1} \oplus A^{j} \mathbb{Z}^{+}\right) \cap\left(n_{2} \oplus A^{j} \mathbb{Z}^{+}\right)=\emptyset$. Thus, every non-negative integer $k$ can uniquely be written as $k=r A^{-j} \oplus s$, where $r \in \mathbb{Z}^{+}, s \in \mathcal{N}_{j}$. 
We now show that the quasi-wavelet system $\widetilde{\mathcal{F}}(\widetilde{\psi}, j, k)$ given by $(42)$ is invariant under translations by $k, k \in \mathbb{Z}^{+}$. In fact

$$
\mathrm{T}_{k} \widetilde{\psi}_{j, n}(x)=\widetilde{\psi}_{j, 0}(x \ominus k)=A^{j} \psi\left(A^{j}(x \ominus k)\right)=\widetilde{\psi}_{j, k}, \quad \text { if } j<0,
$$

and for $j \geq 0, n \in \mathcal{N}_{j}$, we have

$$
\begin{aligned}
\mathrm{T}_{k} \widetilde{\psi}_{j, n}(x) & =\widetilde{\psi}_{j, n}(x \ominus k) \\
& =\psi_{j, d}(x \ominus k) \\
& =A^{j / 2} \psi\left(A^{j}(x \ominus k) \ominus n\right) \\
& =A^{j / 2} \psi\left(A^{j} x \ominus\left(A^{j} k \oplus n\right)\right) \\
& =\psi_{j, k A^{j} \oplus n}(x) .
\end{aligned}
$$

Therefore, the quasi-wavelet system can also be represented as Suppose that $\Lambda=\left\{(\mathfrak{j}, \mathfrak{n}): \mathbf{j} \in \mathbb{Z}, \mathfrak{n} \in \mathcal{N}_{\mathbf{j}}\right\}$. Then, for all $\alpha \in \Lambda$, we set

$$
\widetilde{\mathcal{W}}(\widetilde{\psi}, j, k)=\left\{T_{k} \widetilde{\psi}_{j, n}(x): j \in \mathbb{Z}, k \in \mathbb{Z}^{+}, n \in \mathcal{N}_{j}\right\} .
$$

Suppose that $\Lambda=\left\{(\boldsymbol{j}, \mathfrak{n}): \mathfrak{j} \in \mathbb{Z}, \mathfrak{n} \in \mathcal{N}_{\mathbf{j}}\right\}$. Then, for all $\alpha \in \Lambda$, we set

$$
\varphi_{\alpha}(x)= \begin{cases}A^{j / 2} \psi\left(A^{j} x \ominus n\right), & \text { if } j \geq 0, \\ A^{j} \psi\left(A^{j}(x \ominus n)\right), & \text { if } j<0 .\end{cases}
$$

Therefore, one can easily see that $\varphi_{\alpha} \in \mathrm{L}^{2}\left(\mathbb{R}^{+}\right)$and consequently, the system $\left\{T_{k} \varphi_{\alpha}: k \in \mathbb{Z}^{+}, \alpha \in \Lambda\right\}$ is the quasi-wavelet system $\widetilde{\mathcal{F}}(\widetilde{\psi}, j, k)$. Moreover, the Fourier transform of (46) yields

$$
\widehat{\varphi}_{\alpha}(\xi)= \begin{cases}A^{-j / 2} \widehat{\psi}\left(A^{j} \xi\right) \overline{w_{n}\left(A^{j} \xi\right)}, & \text { if } j \geq 0, \\ \widehat{\psi}\left(A^{j} \xi\right) \overline{w_{n}\left(A^{j} \xi\right)}, & \text { if } j<0 .\end{cases}
$$

Thus, for all $m \in \mathbb{Z}^{+}$, we have

$$
\begin{aligned}
& \sum_{\alpha \in \Lambda}\left|\widehat{\varphi}_{\alpha}(\xi)\right|\left|\widehat{\varphi}_{\alpha}(\xi \oplus m)\right| \\
& \quad=\sum_{j<0}\left|\widehat{\psi}\left(A^{j} \xi\right)\right|\left|\widehat{\psi}\left(A^{j}(\xi \oplus m)\right)\right|+\sum_{j \geq 0} \sum_{n \in \mathcal{N}_{j}} A^{-j / 2}\left|\widehat{\psi}\left(A^{j} \xi\right)\right|\left|\widehat{\psi}\left(A^{j}(\xi \oplus m)\right)\right|
\end{aligned}
$$




$$
=\sum_{j \in \mathbb{Z}}\left|\widehat{\psi}\left(A^{j} \xi\right)\right|\left|\widehat{\psi}\left(A^{j}(\xi \oplus m)\right)\right| \cdot
$$

As a consequence of Theorems 1, 2, 4 and Theorem 8, a necessary condition and two sufficient conditions for wavelet frames on positive half line.

Theorem 9 If the quasi-wavelet system $\left\{\widetilde{\psi}_{j, k}: \mathbf{j} \in \mathbb{Z}, \mathrm{k} \in \mathbb{Z}^{+}\right\}$defined by (42) is a frame for $\mathrm{L}^{2}\left(\mathbb{R}^{+}\right)$with bounds $\mathrm{A}_{7}$ and $\mathrm{B}_{7}$, then

$$
C_{7} \leq \sum_{j \in \mathbb{Z}}\left|\widehat{\psi}\left(A^{j} \xi\right)\right|^{2} \leq D_{7}, \quad \text { a.e. } \xi \in \mathbb{R}^{+}
$$

Theorem 10 Let $\psi \in \mathrm{L}^{1}\left(\mathbb{R}^{+}\right) \cap \mathrm{L}^{2}\left(\mathbb{R}^{+}\right)$. If

$$
\begin{aligned}
& C_{8}=\operatorname{ess} \inf _{\xi \in \mathbb{R}^{+}} \sum_{j \in \mathbb{Z}}\left|\widehat{\psi}\left(A^{j} \xi\right)\right|^{2}-\sum_{n \in \mathbb{N}}\left[\Theta_{\psi}(n) \cdot \Theta_{\psi}(\ominus n)\right]^{1 / 2}>0, \\
& D_{8}=\operatorname{ess} \sup _{\xi \in \mathbb{R}^{+}} \sum_{j \in \mathbb{Z}}\left|\widehat{\psi}\left(A^{j} \xi\right)\right|^{2}+\sum_{n \in \mathbb{N}}\left[\Theta_{\psi}(n) \cdot \Theta_{\psi}(\ominus n)\right]^{1 / 2}<+\infty .
\end{aligned}
$$

Then $\left\{\widetilde{\Psi}_{j, k}(x): j \in \mathbb{Z}, k \in \mathbb{Z}^{+}\right\}$is a wavelet frame for $\mathrm{L}^{2}\left(\mathbb{R}^{+}\right)$with bounds $\mathrm{C}_{8}$ and $\mathrm{D}_{8}$, where

$$
\Theta_{\psi}(n)=\operatorname{ess} \sup \left\{\sum_{j \in \mathbb{Z}}\left|\widehat{\psi}\left(A^{j} \xi\right) \widehat{\psi}\left(A^{j}(\xi \oplus n)\right)\right|: \xi \in \mathbb{R}^{+}\right\} .
$$

Theorem 11 Let $\psi \in \mathrm{L}^{1}\left(\mathbb{R}^{+}\right) \cap \mathrm{L}^{2}\left(\mathbb{R}^{+}\right)$. If

$$
\begin{aligned}
& C_{9}=\operatorname{ess} \inf _{\xi \in \mathbb{R}^{+}}\left\{\sum_{j \in \mathbb{Z}}\left|\widehat{\psi}\left(A^{j} \xi\right)\right|^{2}-\sum_{j \in \mathbb{Z}} \sum_{n \in \mathbb{N}}\left|\widehat{\psi}\left(A^{j} \xi\right) \widehat{\psi}\left(A^{j}(\xi \oplus n)\right)\right|\right\}>0, \\
& D_{9}=\operatorname{ess} \sup _{\xi \in \mathbb{R}^{+}}\left\{\sum_{j \in \mathbb{Z}} \sum_{n \in \mathbb{Z}^{+}}\left|\widehat{\psi}\left(A^{j} \xi\right) \widehat{\psi}\left(A^{j}(\xi \oplus n)\right)\right|\right\}<+\infty .
\end{aligned}
$$

Then $\left\{\widetilde{\Psi}_{j, k}(x): j \in \mathbb{Z}, k \in \mathbb{Z}^{+}\right\}$is a wavelet frame for $\mathrm{L}^{2}\left(\mathbb{R}^{+}\right)$with bounds $\mathrm{C}_{9}$ and $\mathrm{D}_{9}$. 


\section{References}

[1] Abdullah, Affine and quasi-sffine frames on positive half line, J. Math. Ext., 10 (3) (2016), 47-61.

[2] A. Ahmadi, A. A. Hemmat and R. R. Tousi, Shift-invariant spaces for local fields, Int. J. Wavelets Multiresolut. Inf. Process., 9 (3) (2011), 417426.

[3] S. Albeverio, S. Evdokimov, M. Skopina, p-adic multiresolution analysis and wavelet frames, J. Fourier Anal. Appl., 16 (2010), 693-714.

[4] A. Aldroubi, C. Cabrelli, C. Heil, K. Kornelson and U. Molter, Invariance of a shift-invariant space, J. Fourier Anal. Appl., 16 (2010), 60-75.

[5] M. Anastasio, C. Cabrelli and V. Paternostro, Extra invariance of shiftinvariant spaces on LCA groups, J. Math. Anal. Appl., 370 (2010), 530537.

[6] B. Behera, Shift-invariant subspaces and wavelets on local fields, Acta Math. Hungar., 148 (2016), 157-173.

[7] C. de-Boor, R.A. De-Vore, and A. Ron, The structure of finitely generated shift-invariant spaces in $\mathrm{L}^{2}\left(\mathbb{R}^{\mathrm{d}}\right)$, J. Funct. Anal., 119 (1994) 37-78.

[8] M. Bownik, The structure of shift-invariant subspaces of $\mathrm{L}^{2}\left(\mathbb{R}^{n}\right)$, J. Funct. Anal., 177 (2000), 282-309.

[9] C. Cabrelli and V. Paternostro, Shift-invariant spaces on LCA groups, $J$. Funct. Anal., 258 (2010), 2034-2059.

[10] O. Christensen, An Introduction to Frames and Riesz Bases, Second Edition, Birkhäuser, Boston, 2016.

[11] B. Currey, A. Mayeli and V. Oussa, Characterization of shift-invariant spaces on a class of nilpotent Lie groups with applications, J. Fourier Anal. Appl., 20 (2014), 384-400.

[12] R. J. Duffin and A. C. Shaeffer, A class of nonharmonic Fourier series, Trans. Amer. Math. Soc., 72 (1952), 341-366.

[13] Y. A. Farkov, Constructions of MRA-based wavelets and frames in Walsh analysis, Poincare J. Anal. Appl., 2 (2015), 13-36. 
[14] B. I. Golubov, A. V. Efimov and V. A. Skvortsov (1991), Walsh Series and Transforms: Theory and Applications, (Kluwer, Dordrecht).

[15] H. Helson, Lectures on Invariant Subspaces, Academic Press, London, 1964.

[16] S. V. Kozyrev, Wavelet analysis as a p-adic spectral analysis, Izv. Akad. Nauk, Ser. Mat., 66 (2002), 149-158.

[17] A. Yu. Khrennikov and V. M. Shelkovich, An infinite family of p-adic non-Haar wavelet bases and pseudo-differential operators, p-Adic Numb. Ultrametr. Anal. Appl., 3 (2009) 204-216.

[18] S. V. Kozyrev, p-adic pseudodifferential operators and p-adic wavelets, Theor. Math. Phys., 138 (2004), 1-42.

[19] D. Labate, A unified characterization of reproducing systems generated by a finite family, J. Geom. Anal., 12 (2002), 469-491.

[20] D. Li and T. Qian, Sufficient conditions for shift-invariant systems to be frames in $\mathrm{L}^{2}\left(\mathbb{R}^{\mathrm{n}}\right)$, Acta Math. Sinica, English Series., 29 (8) (2013), $1629-1636$.

[21] D. Li G. Wu and X. Yang, Unified conditions for wavelet frames, Georgian Math. J., 18 (2011), 761-776.

[22] P. Manchanda and V. Sharma, Construction of vector valued wavelet packets on $\mathbb{R}^{+}$using Walsh-Fourier transform. Indian J. Pure Appl. Math., 45 (2014), 539-553.

[23] S. Pilipović and S. Simić, Construction of frames for shift-invariant spaces, J. Funct. Spaces Appl., (2013) Article ID. 163814, 7 pages.

[24] R. Radha and N.S. Kumar, Shift-invariant subspaces on compact groups, Bull. Sci. Math., 137 (2013), 485-497.

[25] A. Ron and Z. Shen, Frames and stable bases for shift-invariant subspaces of $\mathrm{L}^{2}\left(\mathbb{R}^{\mathrm{d}}\right)$, Canad. J. Math., 47 (1995), 1051-1094.

[26] F. Schipp, W. R. Wade and P. Simon (1990), Walsh Series: An Introduction to Dyadic Harmonic Analysis, (Adam Hilger, Bristol and New York). 
[27] F. A. Shah, Gabor frames on a half-line, J. Contemp. Math. Anal., 47 (5) (2012), 251-260.

[28] Y. Zhang, Walsh Shift-Invariant Sequences and p-adic Nonhomogeneous Dual Wavelet Frames in $\mathrm{L}^{2}\left(\mathbb{R}^{+}\right)$, Results Math., 74111 (2019), 26 pp.

Received: August 28, 2020 